\title{
Hydraulics and Pneumatics: Operational Characteristics and Control for Modern Industry Applications
}

\author{
M. Papoutsidakis \\ Dept. of Industrial \\ Design and Production \\ Engineering \\ University of West Attica, \\ Athens, Greece
}

\author{
A. Chatzopoulos \\ Dept. of Industrial \\ Design and Production \\ Engineering \\ University of West Attica, \\ Athens, Greece
}

\author{
D. Papachristos \\ Dept. of Industrial \\ Design and Production \\ Engineering \\ University of West Attica, \\ Athens, Greece
}

\author{
C. Drosos \\ Dept. of Industrial \\ Design and Production \\ Engineering \\ University of West Attica, \\ Athens, Greece
}

\begin{abstract}
The research of ways to obtain higher and higher pressures that are impossible to be gained with human power is a challenge that starts way back in the history of mankind. In modern days, technological steps in this direction take place almost daily to improve human life, both directly and indirectly. The two main methods to gain high pressures are through hydraulic and pneumatic systems, which have a large application in nearly every field of engineering. Hydraulic systems work according to the principle that the pressure exerted by a confined liquid is transmitted to the internal face of its container. Applying this principle in a hydraulic system, the force exerted by a liquid can be transferred to a piston to generate power. Pneumatic systems, instead, use compressed air to transmit and control energy.
\end{abstract}

This paper will present a bibliographic research on the main applications of these two systems, analysing their components and operation. Moreover, deepening on pneumatic systems, this study will focus on one of the most modern pneumatic machine in food packaging: the Tetra Pak's A6. This machine is the first and only device in the world capable of packing UHT milk in one-litre aseptic carton bottles. The 10 steps required by the machine to fulfil its function, will be examined in detail, alongside with its connections, sensors and controllers.

\section{Keywords}

Hydraulics, Pneumatics, Food Packaging.

\section{INTRODUCTION}

From the beginning of the world, people always tend to reach higher pressures that they cannot provide by human power. Hydraulic and pneumatic systems are based on the processes that can provide higher pressures in the easiest ways.

Hydraulic power idea is based on pressurized fluid in order to produce power. Today most of the processes done in high pressures is provided by hydraulic systems. The history of hydraulic systems can be initiated with the usage of water as a pressure source. The first application of hydraulic system is based on irrigation and appeared in Mesopotamia and ancient Egypt since 6th millennium BC. Also, in everyday life, Imperial Rome society used water power in order to produce flour, saw stone and timber, while Britain society were using hydraulic systems in mining processes. In time, hydraulic mining is developed and used in California Gold Rush. In 1648 , hydraulic evolved into a science with the discovery of "Pascal's Law" by Blaise Pascal. After the discovery of Pascal's Law, Daniel Bernoulli took the hydraulic science one step further and came up the idea of "Bernoulli's Principle" which describes the behaviour of fluid under different condition of flow and height. The first practical hydraulically operated machine is "The Hydraulic Press", that was invented in 1795 by Joseph Bramah and is based on Pascal Law. In late 19th century, William George Armstrong, affected by the studies of Braham, developed the "Hydraulically Operated Crane" for the wharf in Newcastle-on-Tyne. Moreover, Hydraulically Operated Crane was installed for wharves and railway yards in other places in UK. Armstrong's Elswick Hydraulic Equipment Company manufactured high-pressure hydraulic machinery for the power required in Australia as a central power plant system to supply hydraulically operated coal loading cranes for the Port of Newcastle in Australia. The first public hydraulic power system was operated in Hull, UK in 1876. The high-pressure water to operate hydraulic machine was provided to the individual usage of customers with this system. Today there are many different forms of hydraulic power, most of them generate electricity such as building processes, diggers, cranes, bulldozers and all kinds of heavy equipment vehicles rely on power provided from hydraulic systems. Hydraulic systems are devices that use pressurised fluid in order to drive the machinery and it is made up of many components. Hydraulic pump which can have a power density of up to 10 times that of an electric motor. It is clearly seen that; hydraulic systems provide power in the form of pressurized liquid that cannot be reached by human muscle.

Pneumatic systems are generally used in industrial machines that are powered by compressed air or compressed inert gases. Pneumatic systems are used to convert gases pressure to mechanical power. Practically, the machines work with vacuum and positive gases pressure are categorized in pneumatic systems. The required air is provided to the system by pressurized compressor which stores the air in strengthened steel tank. The first compressors were seen around 3000 B.C. to provide small puffs of air to aid in starting a fire. The origination of pneumatics can be connected with the ancient Greek mathematician Hero of Alexandria in the first century. The study is about pneumatic systems that powered by steam and wind. German physicist Otto von Guericke in 1600's moved pneumatic science a step further and invented a vacuum pump that was utilized with air pressure. In 1800's, pneumatic systems evolved in another level that increases the supplied pressure to higher level by systems. The compound air compressor, which compresses air in a series of cylinders, was first patented in 1829. The compressor technology improved further as the cylinders were cooled by water to improve efficiency in 1872 and this resulted in the invention of water-jacketed cylinders. The most functional development was a pneumatic subway train in New York made by American inventor, Alfred Beach in 1867. The project showed how passengers could be transported 
along a pipe through pneumatic power. Pneumatics also used for transporting letters from one area of the building to another in late 1800 's. This pneumatic system is invented by American John Wanamaker. Another pneumatic system which is pneumatic drill was invented in 1871 by Samuel Ingersoll. Moreover, later in 1890, Charles Brady invented pneumatic-powered hammer. In 19th century, pneumatics was used to power a tunnelling project in Mt. Cenis, located in the Alps. If traditional drilling methods were used the project would last 30 years. Using pneumatic drills, operating on many miles of line, the tunnel was completed in just 14 years and it is opened to traffic in 1871. In middle of the 19th century, pneumatics was started to apply in power applications. Especially, air pressurized hand tools, drillers and locomotives lead to develop pneumatic systems. The 1900 's welcomed the evolution of pneumatics as components were used for the first time in jet engines in the form of centrifugal and axial-flow compressors. In 20th century, further developments as advancements in labour-saving devices in the form of machinery that would assist or reduce the need for manpower appeared. As well as, automatic machinery, tools and control systems developed during this century. Towards the end of the 1960's, the first digitally controlled pneumatic components entered in to the market and helped the revolution of the idea of pneumatic systems in power sector. Today, pneumatic systems have reached the level of modern control elements. Without any doubt that, pneumatics has a great history that proves the technology of pneumatic systems will grow continuously with innovative pneumatic solutions required.

\section{SYSTEM AND COMPONENTS}

\subsection{Hydraulics}

The hydraulic systems are based on the principle of Pascal's Law by French scientist Blaise Pascal which states the pressure exerted anywhere by a confined liquid is transmitted, undiminished in all directions to the internal surface of the container, equally at right angles. The principle allows large forces to be generated with comparatively small forces. In a basic hydraulic system, the force is depending on the parameters of the bore size of cylinder and the pressure supplied by pump to the cylinder. As well as, the speed of the piston is related with the flow rate provided by pump and cylinder area. Hydraulic systems are made up with several interconnected components to transmit and control power through pressurized fluid. The basic components of a fluid power system are:

$\begin{array}{ll}\text { i. } & \text { Reservoir } \\ \text { ii. } & \text { Pumps } \\ \text { iii. } & \text { Strainers and filters } \\ \text { iv. } & \text { Directional Control Valves } \\ \text { v. } & \text { Relief Valve } \\ \text { vi. } & \text { Connectors \& Fittings } \\ \text { vii. } & \text { Cylinder }\end{array}$

vii. Cylinder

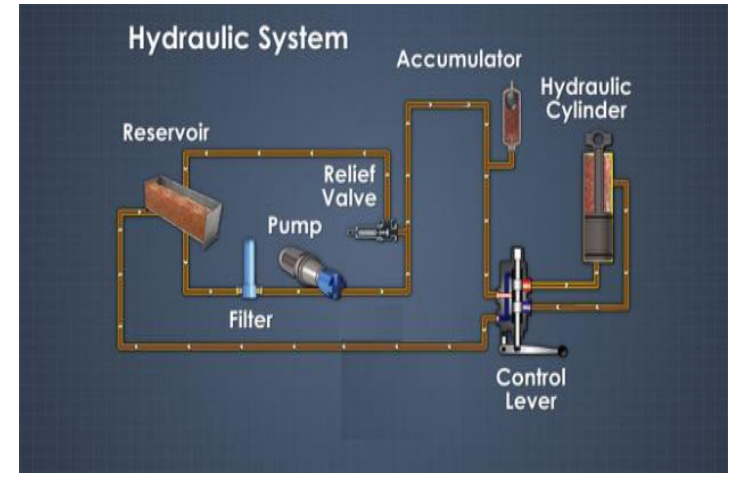

Fig 1

\section{i. $\quad$ Reservoir}

A properly constructed reservoir is a tank to hold oil until the system demands fluid. It is also capable to dissipate heat from the fluid, separate air from the oil and settle out contamination in the oil. The reservoir generally has fluid three times more than the pump output per minute.

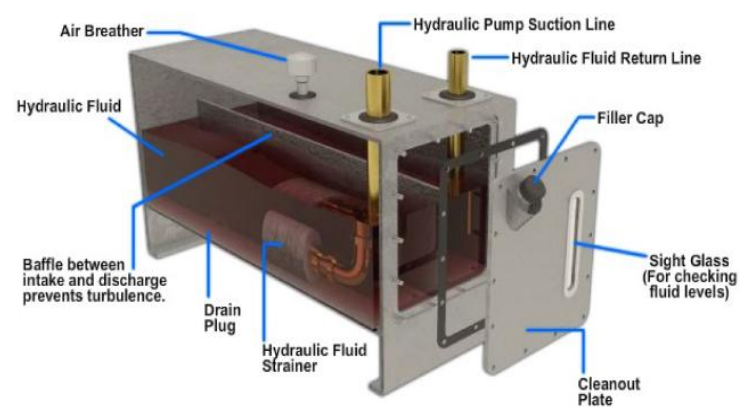

Fig 2

\section{ii. Pumps}

The purpose of a hydraulic pump is supplying a flow of fluid to a hydraulic system. As the pump provides flow, it transmits a force to the fluid. As the fluid flow encounters resistance, this force is changed into pressure. The hydraulic pump is used to force the fluid from the reservoir to rest of the hydraulic circuit by converting mechanical energy into hydraulic energy. The most common two types of pumps are centrifugal pumps and reciprocating pumps.

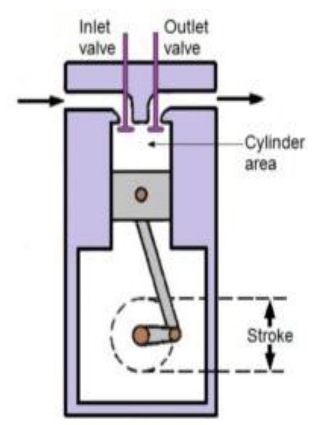

Fig 3.a

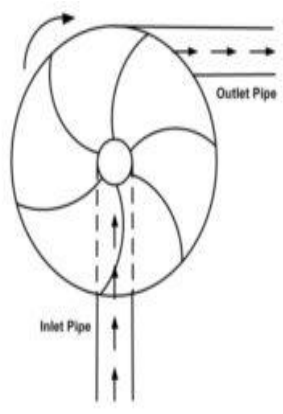

Fig3.b

\section{iii. Strainers and filters}

Strainers are constructed of fine mesh wire screens or of screening elements consisting of specially processed wire of varying thickness wrapped around metal frames. They do not provide as fine a screening action as filters, but they offer less resistance to flow and are used in pump suction lines where pressure drop must be kept to a minimum. On the other hand, the most common device installed in hydraulic systems to prevent foreign particles and contaminations from remaining 
in the system are called filters. They may be located in the reservoir, in the return line, in the pressure line, or in any other location in the system where the system requires protection against impurities.

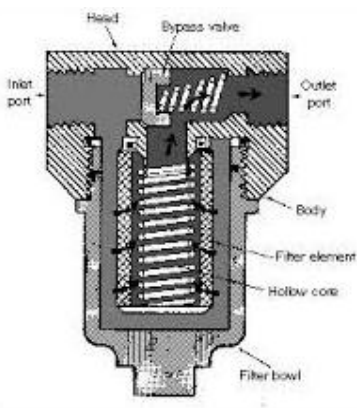

Fig 4.a

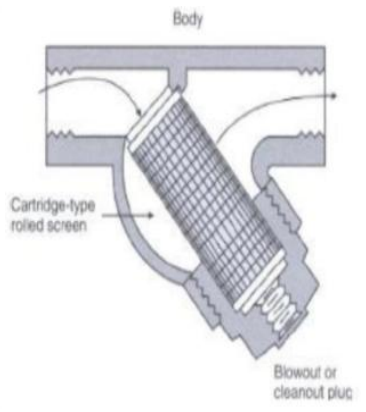

Fig 4.b

\section{iv. Directional Control Valves}

Directional control valves are designed to direct the flow of fluid to a point in a hydraulic system. Also, they may be operated by differences in pressure acting on opposite sides of the valving element, or may be positioned manually, mechanically, or electrically. Often two or more methods of operating the same valve will be used in different phases of its action.

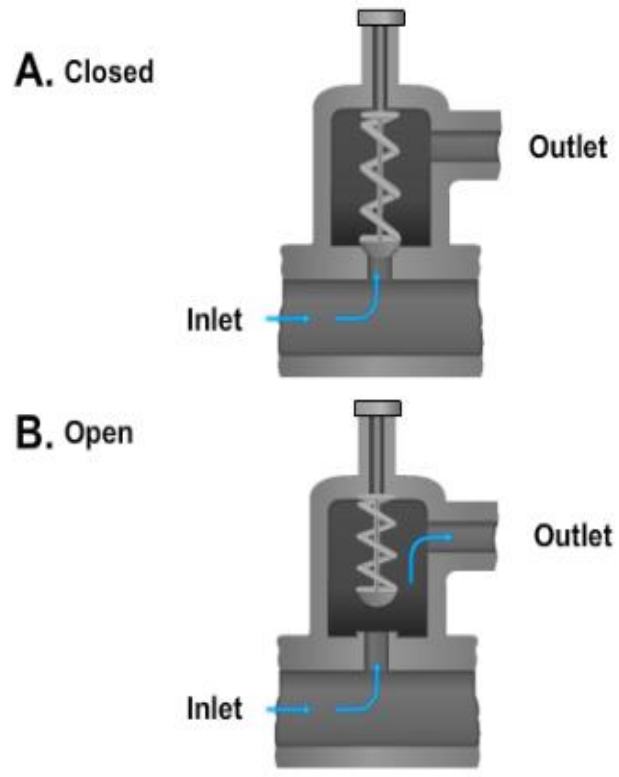

Fig 5

\section{v. Relief Valve}

The safe and efficient operation of hydraulic circuits, systems components, and related equipment require a type of controlling pressure. There are many types of automatic pressure control valves. Some of them only provide an escape for pressure that exceeds the set pressure, some only reduce the pressure to a lower pressure system or subsystem, and some keep the pressure in a system within a required.

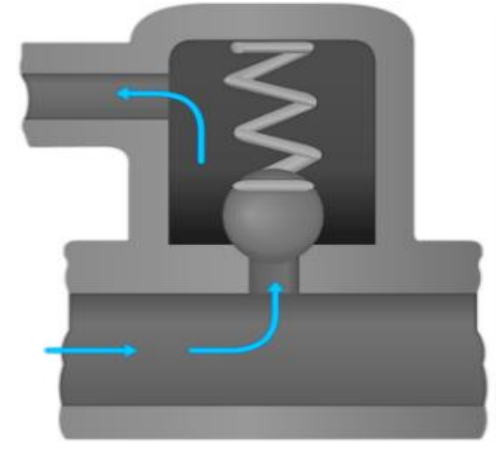

Fig 6

\section{vi. Connectors \& Fittings}

There are many types of connectors and fittings required for a fluid power system. Fittings are used to connect the units of a hydraulic system, including the individual sections of a circulatory system. The type of connector or fitting depends upon the type of circulatory system (pipe, tubing, or flexible hose), the fluid medium, and the maximum operating pressure of the system. Some of the most common types of connectors are threaded connectors, flared connectors, flexible hose couplings, and reusable fittings. Threaded connectors are used in low pressure pipe systems. The connectors are made with standard pipe threads cut on the inside surface of the connector. The end of the pipe is threaded on the outside for connecting with the connector. Standard pipe threads are tapered slightly to ensure a tight connection.

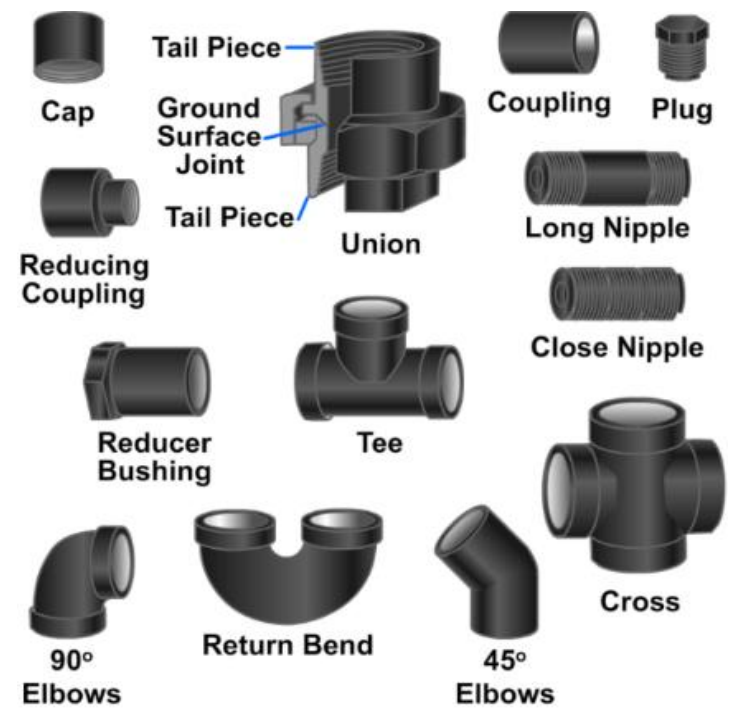

Fig 7

vii. Cylinder

A hydraulic cylinder is a mechanism that converts energy stored in the hydraulic fluid into a force used to move the cylinder in a linear direction. Also, it produces a force resulting in linear motion, whereas a fluid motor produces a torque resulting in rotary motion. It has many applications and can be either single acting or double acting. 


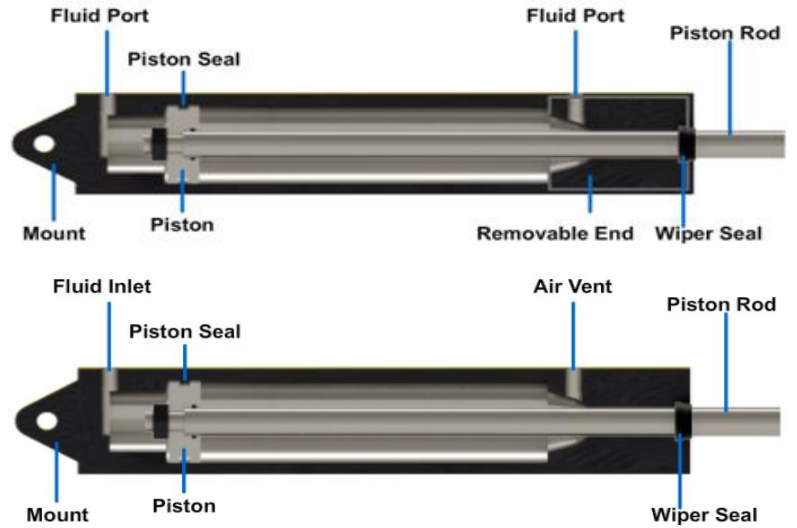

Fig 8

\subsubsection{Operation of Hydraulics}

While control valve is in neutral position, the pump flow passes through the valve and back to the reservoir. When the valve is shifted, oil is directed to the piston side of the cylinder and causes the piston to move while extending the rod. If the valve is returned to neutral, the oil is trapped in the cylinder, holding it in a fixed position, while pump flow is returned to the reservoir. Shifting the valve in the opposite direction permits the oil to pass through the valve back to the reservoir. The relief valve limits the system pressure to a certain amount. Relief valves are commonly incorporated into the directional control valve.

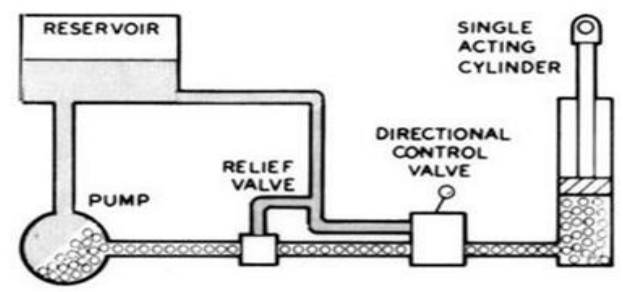

Fig 9

A hydraulic system with a double acting cylinder and a 4-way valve is different from the single acting cylinder system where the cylinder can exert force in both directions. When the control valve is in neutral, flow is returned to the reservoir. When shifted in one direction, oil is directed to the piston side of the cylinder, causing the cylinder to extend. Oil from the rod side passes through the valve back in order to flow to reservoir. If the valve is shifted to neutral, oil in the cylinder is trapped, holding it in a fixed position. When the valve is shifted in the opposite position, oil is directed to the rod side of the cylinder, causing the cylinder to retract. Oil from the piston side passes through the valve back to the reservoir.

\subsection{Pneumatic}

The pneumatic systems are based on the system that uses compressed air to transmit and control energy. Most pneumatic systems rely on a constant supply of compressed air to utilize. This pressure is provided by an air compressor. The compressor takes air from the atmosphere and stores it in a high pressure tank called a receiver. This compressed air is then supplied to the system through a series of pipes and valves. The main components of a pneumatic system are:

$$
\begin{array}{cl}
\text { i. } & \text { Control Valve } \\
\text { ii. } & \text { Cylinder } \\
\text { iii. } & \text { Compressor } \\
\text { iv. } & \text { Sensor } \\
\text { v. } & \text { Filter } \\
\text { vi. } & \text { Reservoir (Receiver) } \\
\text { vii. } &
\end{array}
$$

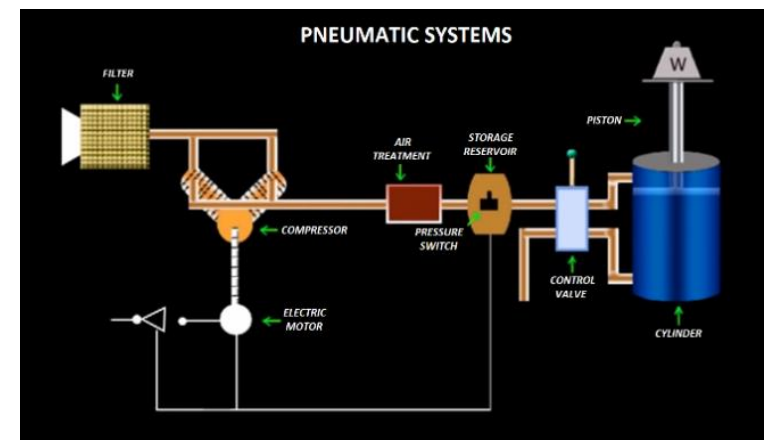

Fig 11

\section{i. Control Valve}

Valves provide direction control of air, arrange the flow rate and pressure air. The main function of the control valve is maintaining constant downstream pressure in the air line. Due to the high velocity of the compressed air flow, there is flowdependent pressure drop between the reservoir and load. Hence the pressure in the reservoir is always kept higher than the system pressure. Non-return valves allow air to flow in one direction only. When air flows in the opposite direction, the valves will close. Flow control valves control the speed of air flow into or out of a pneumatic circuit or component. Flow control is adjusted using the needle valve. A flow control valve is formed by a non-return valve so air is regulated in one direction only, and flows freely in the other direction. Directional control valves ensure the flow of air between air ports by opening, closing and switching their internal connections. Their classification is determined by the number of ports, the number of switching positions, the normal position of the valve and its method of operation.

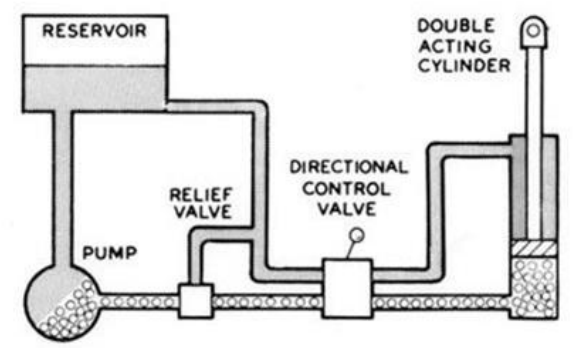




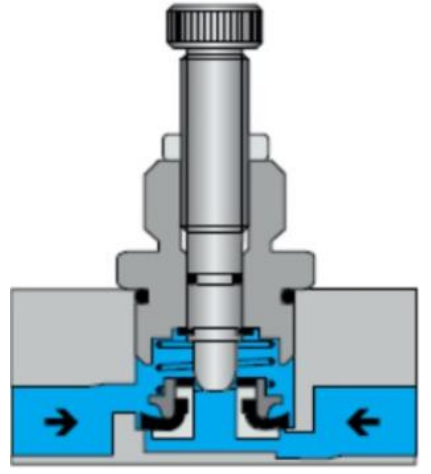

Fig 12

\section{ii. Cylinder}

Single acting cylinder has only one entrance that allows compressed air to flow through. Therefore, it can only produce thrust in one direction. The piston rod is moved in the opposite direction by an internal spring, or by the external force provided by mechanical movement or weight of a load. Single acting cylinders are used in stamping, printing, moving materials.

In double acting cylinder, air pressure is applied alternately to the relative surface of the piston, producing a pushing force and a retracting force.

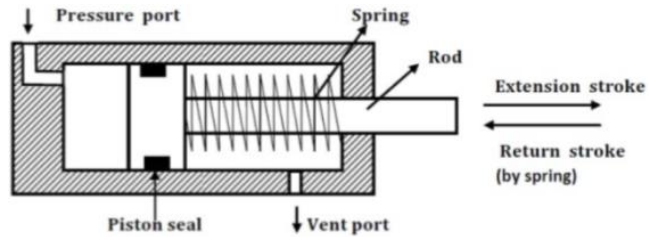

Fig 13
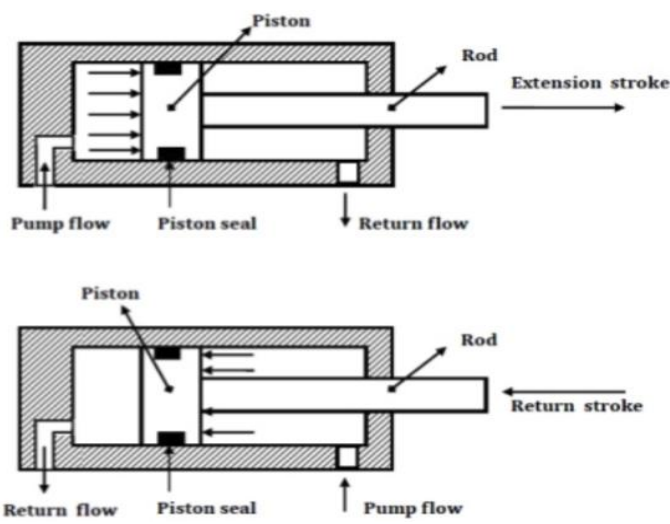

Fig 14

\section{iii. Compressor}

Air compressor converts the mechanical energy of an electric or combustion motor into the potential energy of compressed air. The compressor increases the pressure of fresh air drawn from the atmosphere. External power supply called motor is used to drive the compressor. There are several types of compressors which are used in the compressed air systems. Compressors generates compressed air which is selected on the basis of desired maximum delivery pressure and the required flow rate of the air. A single central compressor can supply various pneumatic components with compressed air, which is transported through pipes from the cylinder to the pneumatic components. The types of compressors are, piston or reciprocating compressors, rotary compressors, centrifugal compressors and axial flow compressors.

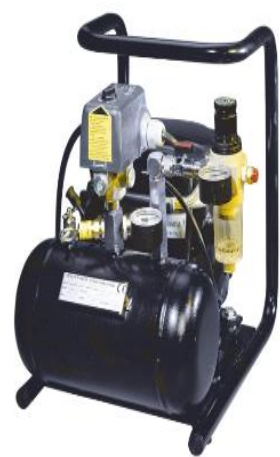

Fig 15

\section{iv. Sensors}

There are several types of sensors in pneumatic systems; however, the main sensors of pneumatic systems can be categorized as displacement and pressure sensors. Pneumatic sensors are used to measure the displacement and to sense the proximity of an object close to it. The displacement and proximity are transformed into change in air pressure. Figure16 shows a schematic of a sensor about its construction and working process. It is made up of three ports. Low pressure air is allowed to escape through port A. In the absence of any obstacle / object, this low pressure air escapes and result in, reduction of the pressure in the port $\mathrm{B}$. However when an object obstructs the low pressure air (Port A), there is rise in pressure in output port $\mathrm{B}$. This rise in pressure is calibrated to measure the displacement or to trigger a switch. These sensors are used in robotics, pneumatics and for tooling in CNC machine tools.

Pressure sensors help to control a high-speed manufacturing system or monitoring various inlet and outlet pressures within an air compressor to determine performance and efficiency. Pressure-sensing devices can be categorized as either a switch or transducer. They enable pneumatic systems to move precisely and apply the required amount of force or torque. A typical pressure switch consists of a contact driven by a diaphragm or piston, which is pushed in one direction by the fluid being sensed and a bias spring that pushes in the opposite direction. The switch's contact changes state when the pressure on the switch's inlet side rises above the pressure exerted by the bias spring on the other side. Conversely, the switch returns to its original state once the inlet pressure falls below a predetermined threshold. Due to a phenomenon known as hysteresis, that threshold may or may not be the same as its actuation level. In some cases, hysteresis can be used to one's advantage when attempting to stabilize pneumatic systems.
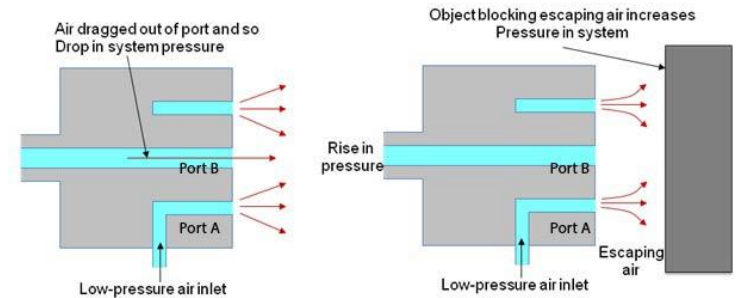

Fig 16.a 


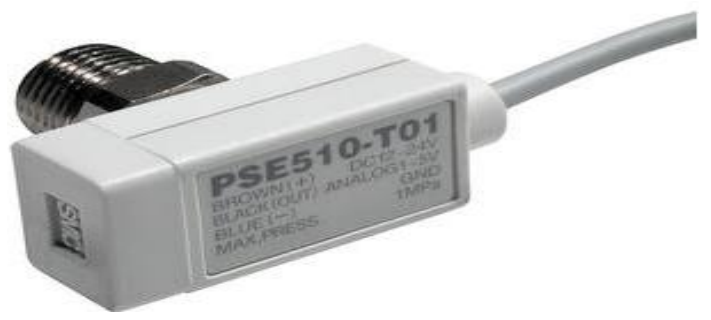

Fig 16.b

\section{v. $\quad$ Filter}

A pneumatic filter is a device which removes contaminants from a compressed air stream. While blocking contaminant particles the membrane of filter allows air to pass through to a venturi. A pneumatic filter should be placed as the first component at the inlet of air circuits. Filter usually combined with components that regulates the pressure of air and adds lubricants for moving parts in the circuit.

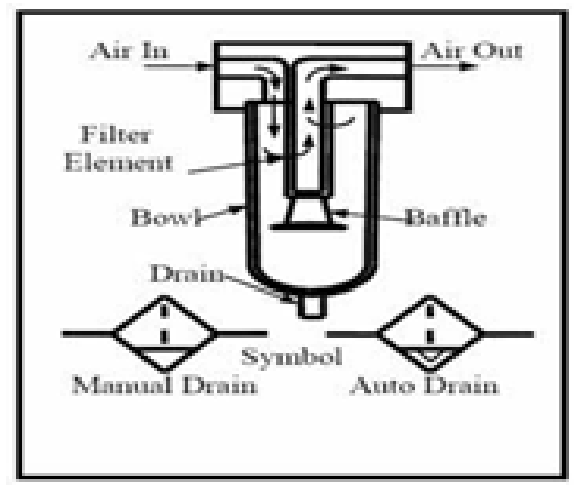

Fig 17

\section{vi. Reservoir (Receiver)}

The pneumatic system needs continuous supply of air, but the air is compressed slowly in the compressor, so this compressed air has to be stored. The compressed air is stored in an air receiver. The air receiver smoothens the flow from the compressor. It also helps the air to cool and condense the moisture. The air receiver should be large enough to hold all the air delivered by the compressor. The pressure in the receiver is greater than the operating system pressure to compensate pressure loss in the pipes. Also the large surface area of the receiver helps dissipating of the heat from the compressed air. Generally the size of receiver depends on, delivery volume of compressor, air consumption, pipeline network, type and nature of on-off regulation and permissible pressure difference in the pipelines.

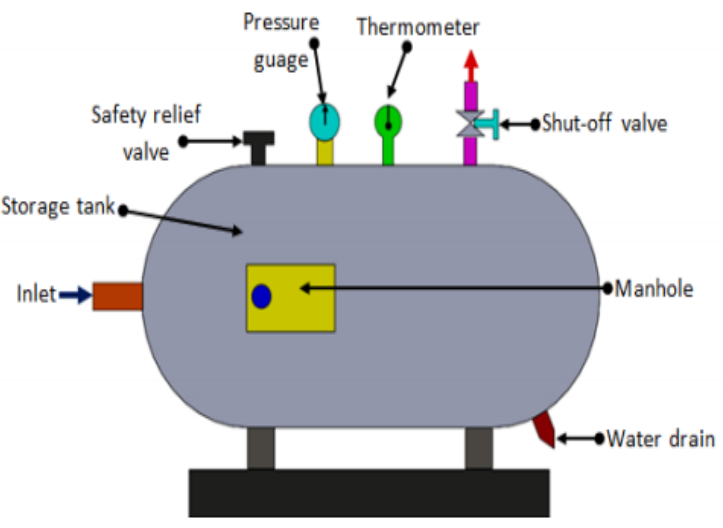

Fig 18

\subsubsection{Operation of pneumatics}

In pneumatic system, as an initiation the air is obtained by the filter from atmosphere. Atmospheric air is contaminated with dust, smoke and is humid. In filter, air is separated by its impurities. External particles can cause wear on system components and presence of moisture may cause corrosion. The filter provides pure air to the compressor which is driven by electric motor. In compressor, clean air is pressurized to the high level of pressure. Air compressor converts the mechanical energy of an electric or combustion motor into the potential energy of compressed air. Furthermore, during compression operation, air temperature increases. Therefore, cooler is used to reduce the temperature of the compressed air. The system of pneumatic circuit requires continuous air supply. The reservoir stores compresses air that arrived from compressor through pipes and provide pressurized air when needed. Control valve is used to decide in which way the load will be directed by system. If the control valve is in neutral position, no air flows through the cylinder. If it is switched, the air flows through the cylinder and directs load to up or down depending on the position of control valve and the piston.

\section{MODERN PNEUMATIC SYSTEM FOR FOOD PACKAGING}

\subsection{Parts \& Operation Description}

The A6 is Tetra Pak's latest released aseptic machine system. It is a machine used for packing UHT milk in one-litre aseptic carton bottles. The carton bottle is made of cardboard and has a moulded plastic top. It has a large screw cap that makes pouring easy without splashing. The machine is the first aseptic carton bottle in the world and it was created as a response to the PET bottle being sold in southern Europe. Therefore, the target was warm countries like Portugal, Spain and Italy, where the cost of refrigerated transport and storage is expensive.

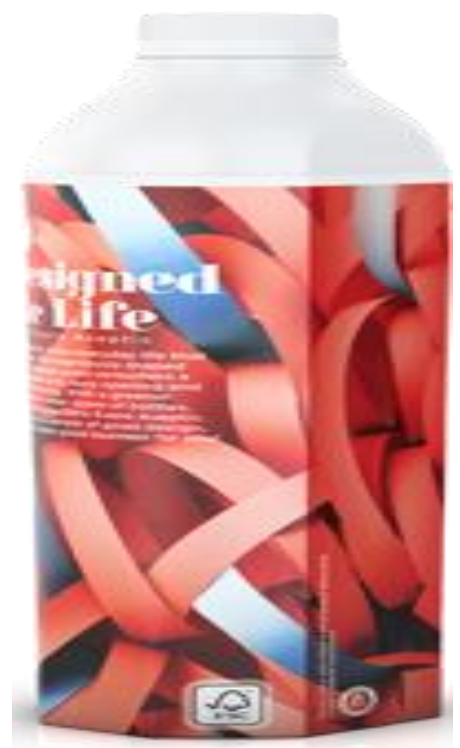

Fig 19

The A6 is a two-sided machine where each machine side produces five thousands packages per hour. The machine production uses the following physical material: packaging material, strip, plastic granulate, capped necks, and a product. 
The A6 machine is Tetra Pak's largest machine system, it has two floors and is over 15 meters long, see Figure 2. The A6 machine system is constructed of 11 different modules produced at various module suppliers where they are also test ran, the modules are thereafter shipped to Tetra Pak Carton Bottle in Lund where they are docked together and further tests are performed before the whole machine is shipped to the customer.

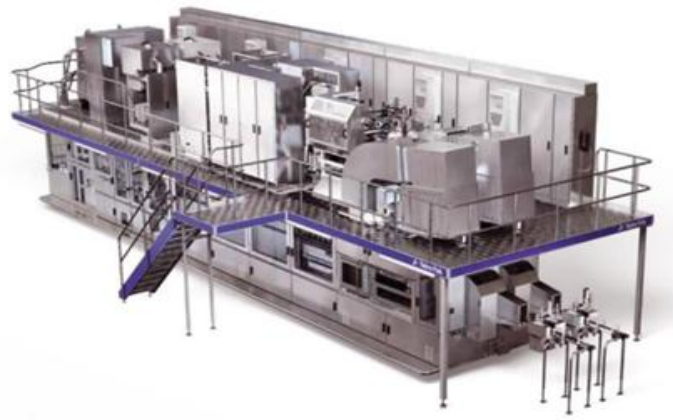

Fig 20

$\begin{aligned} \text { 3.1.1 } & \text { Machine Flow } \\ \text { i. } & \text { Package material feeding and Strip application } \\ \text { ii. } & \text { Cut into sheets } \\ \text { iii. } & \text { Sleeve forming } \\ \text { iv. } & \text { Top Moulding } \\ \text { v. } & \text { Transport into SFS } \\ \text { vi. } & \text { 3 step sterilization } \\ \text { vii. } & \text { Four stations filling } \\ \text { viii. } & \text { Sealing } \\ \text { ix. } & \text { Fold and form } \\ \text { x. } & \text { Date marking }\end{aligned}$

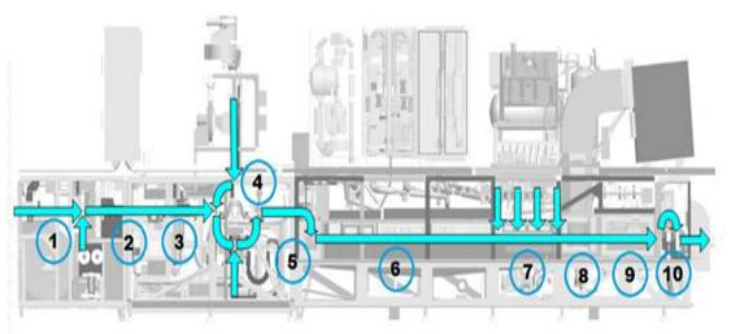

Fig 21

\section{i. $\quad$ Package material feeding and Strip application}

The material flow starts in the first module, called ASU \& Cutting. The module supplies the machine with packaging material and strip. Both materials are sealed together by melting one edge of the packaging material. The strip is applied to protect the product, preventing bacteria in the fibers to reach the product.

\section{ii. Cut into sheets}

The package material with the applied strip is cut into sheets.

\section{iii. Sleeve forming}

The sheet is formed to a sleeve by squeezing it around a mandrel and sealed the sides using induction.

\section{iv. Top Moulding}

The sleeve is pulled off from the forming mandrel onto a mandrel wheel. On the mandrel a capped neck is placed and prepared. The mandrel wheel indexes once and the sleeve is now pointing downwards, from below a so called hotrunner is lifted up to the sleeve which moulds a plastic top on the sleeve. The moulding forms a plastic cover between the capped neck and the sleeve, thereby binding the two together and creating a Ready-to-Fill (RTF) package.

\section{v. Transport into SFS}

After one more indexing the RTF pack is transferred into the aseptic chamber and positioned bottom-up. The package has at this point been transferred from the RTF part of the machine to the SFS, Sterilize-Fill-Seal part.

\section{vi. 3 step sterilization}

Each RTF pack goes through a three stage sterilization process. During the first stage the RTF pack is preheated to prevent condensation during the second stage. At the second stage the RTF pack is gassed and sterilized with hydrogen peroxide. In the third stage the hydrogen peroxide gas is vented away and neutralized by a catalyst converting the gas to water and oxygen. Once the hydrogen peroxide has been vented away then non-sterile air must be prevented from reaching the inside of the RTF pack until it has been filled and sealed. Sterile air is therefore blown downwards from the top of the aseptic chamber, pressing the non-sterile air downwards. A virtual barrier is formed where the upper part of the chamber is sterile and the lower part is non-sterile.

\section{vii. Four stations filling}

Once the sleeve has been vented empty of hydrogen peroxide, it is ready to be filled. Four stations are used to fill the RTF package with one litre of a product. The partial filling is to reduce foaming, as excess foaming may prevent the RTF pack from being aseptically sealed.

\section{viii. Sealing}

The filled but open container is indexed forward, with a motion profile that does not spill the milk. At the sealing station the open sleeve is squeezed together and sealed using induction heating, thus creating an aseptic sealed package.

\section{ix. Fold and form}

At the end of the aseptic chamber the bottom of the filled pack is formed. A cylinder presses the bottom down so that two flaps are formed. The plastic on the inside of the flaps is melted with hot air and finally the flaps are folded against the underside of the bottle. The aseptic carton bottle is now complete.

\section{x. Date marking}

The aseptic carton bottle is now complete, and an arm lifts the bottle out of the aseptic chamber and places it upright on a conveyor belt. The bottle is now transported out of the machine, through the date marking equipment and to the distribution equipment where it will be wrapped in film or put in cardboard boxes.

\subsection{Connectors}

Permanent joints are welded (Figure-22). Where disconnection is required, the pipe connection is in the form of a threaded union with a male end and a retained nut with a joint ring in between, or a clamped union with a joint ring (Figure 23).

The union permits disconnection without disturbing other pipe-work. This type of joint is therefore used to connect 
process equipment, instruments, etc. that need to be removed for cleaning, repair or replacement.

$\begin{array}{ll}\text { i. } & \text { Tees } \\ \text { ii. } & \text { Reducers } \\ \text { iii. } & \text { Bends }\end{array}$

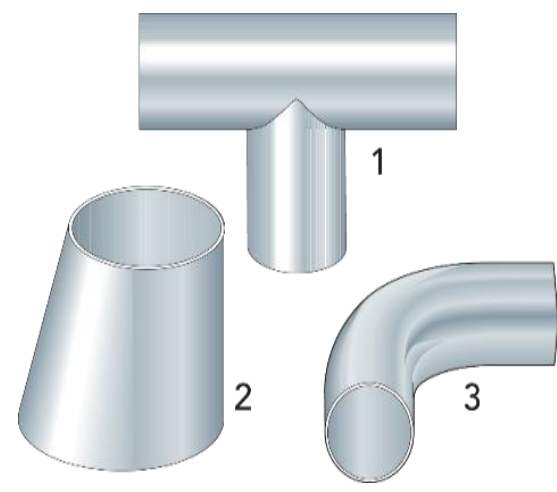

Fig 22

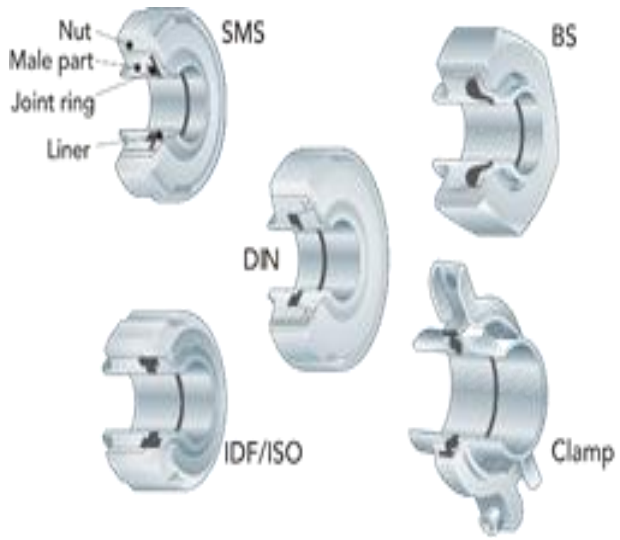

Fig 23 :"Dairy unions of different standards"

All unions must be tightened firmly to prevent liquid from leaking out or air from being sucked into the system and causing problems in downstream parts of the process.

\subsubsection{Special Pipe Fittings}

Sight glasses are fitted in the line where a visual check of the product is required. Bends with instrument connections are used for fitting instruments like thermometers and gauges. The sensor should be directed against the flow to make readings as accurate as possible. Instrument connections can also be provided with welding special bosses directly onto the pipe during installation.

\subsubsection{Sampling Devices}

Sampling devices need to be installed at strategic points in the plant to collect product samples for analysis. For quality control, such as determining the fat content of milk and the $\mathrm{pH}$ value of cultured products, the samples can be collected from a sampling cock (Figure-24).

For hygienic quality tests, the sampling method must preclude any risk of contamination from outside the pipe. A sampling plug can therefore be used. This plug, shown in (Figure-25), has a rubber bung at the bottom. The plug is first removed and all parts that could contaminate the sample are sterilized, after which the needle of a hypodermic syringe is inserted through the bung into the product, and a sample is withdrawn.

The aseptic sampling valve (Figure-26) consists of three parts, a valve body, a valve head and a membrane. The rubber membrane is placed on the stem of the valve head and works as a stretchable plug. The aseptic sampling valve is designed for sterilization before and after each sampling.

The manual valve is opened by rotating a handle or by activating a lever. The stem and the membrane are then retracted, allowing liquid to pass.

Using the reverse procedure, the built-in spring closes the valve and keeps the channel between the hose pieces open for sterilization.

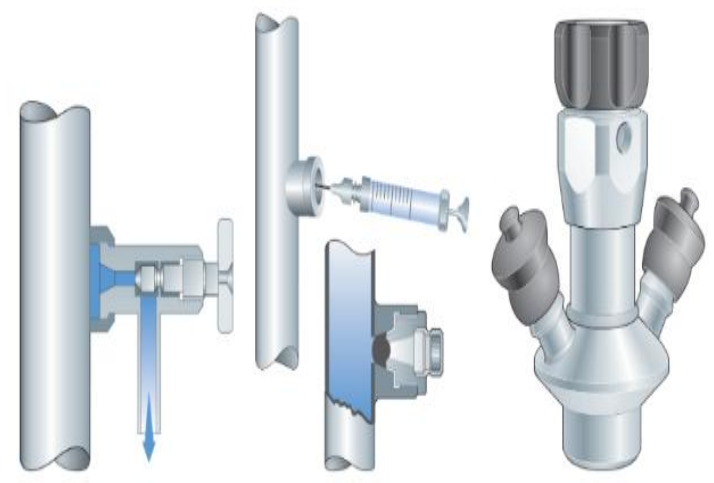

Fig 24

\subsection{PID Controllers}

\subsubsection{Figures \& Block Diagram}

In automated industrial processes, it is always essential to keep the process variables such as temperature, flow rate, system pressure, fluid level, etc. at the desired value for safety and economical operation. The control can be achieved by using control electronics or by pneumatic process control. The pneumatic systems are quite popular because they are safe. There are many different types of pneumatic controller one of them is the PID Controller.

The "Proportional-Integral-Derivative controller" (PID controller) is a control loop feedback mechanism that continuously calculates an error value $\mathrm{e}(\mathrm{t})$ as the difference between a desired setpoint (SP) and a measured process variable (PV) and applies a correction based on proportional, integral, and derivative terms (denoted P, I, and D respectively), hence the name. This type of control system is called closed loop control system.

The characteristic of the PID controller is the ability to use the three control terms of proportional, integral and derivative influence on the controller output to apply accurate and optimal control.

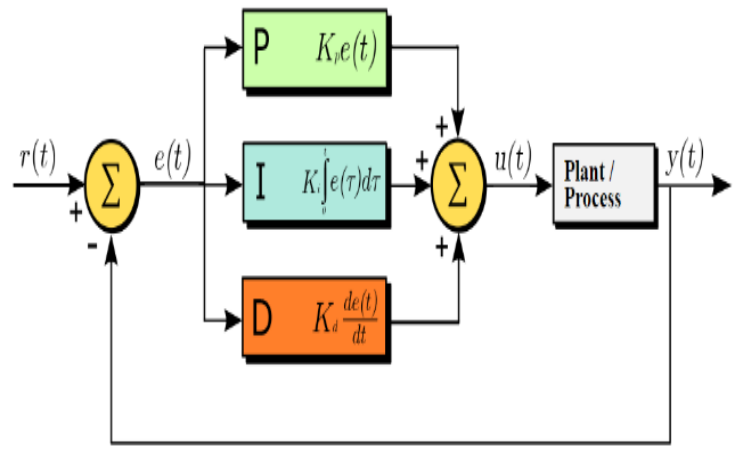

Fig 25 
The block diagram shows the principles of how these terms are generated and applied.

It shows a PID controller, which continuously calculates an error value $e(t)$ as the difference between a desired setpoint $\mathrm{SP}=\mathrm{r}(\mathrm{t})$ and a measured process variable $\mathrm{PV}=\mathrm{y}(\mathrm{t})$, and applies a correction based on proportional, integral, and derivative terms.

The controller attempts to minimize the error over time by adjustment of a control variable $\mathrm{u}(\mathrm{t})$ such as the opening of a control valve, to a new value determined by a weighted sum of the control terms. In this model:

Term $\mathrm{P}$ is proportional to the current value of the $\mathrm{SP}-\mathrm{PV}$ error $\mathrm{e}(\mathrm{t})$. For example, if the error is large and positive, the control output will be proportionately large and positive, taking into account the gain factor " $\mathrm{K}$ ". Using proportional control alone in a process with compensation such as temperature control, will result in an error between the setpoint and the actual process value, because it requires an error to generate the proportional response. If there is no error, there is no corrective response.

Term I accounts for past values of the SP - PV error and integrates them over time to produce the I term. For example, if there is a residual SP-PV error after the application of proportional control, the integral term seeks to eliminate the residual error by adding a control effect due to the historic cumulative value of the error. When the error is eliminated, the integral term will cease to grow. This will result in the proportional effect diminishing as the error decreases, but this is compensated for by the growing integral effect.

Term D is a best estimate of the future trend of the SP - PV error, based on its current rate of change. It is sometimes called "anticipatory control", as it is effectively seeking to reduce the effect of the SP-PV error by exerting a control influence generated by the rate of error change. The more rapid the change, the greater the controlling or dampening effect.

\subsubsection{Tuning}

The balance of these effects is achieved by "loop tuning" to produce the optimal control function. The tuning constants are shown below as "K" and must be derived for each control application, as they depend on the response characteristics of the complete loop external to the controller. These are dependent on the behaviour of the measuring sensor, the final control element (such as a control valve), any control signal delays and the process itself. Approximate values of constants can usually be initially entered knowing the type of application, but they are normally refined, or tuned, by "bumping" the process in practice by introducing a setpoint change and observing the system response.

\section{CONCLUSION}

According to this study, both hydraulic systems and pneumatic systems are the ways of fluid power in order to provide great pressure. There are a lot of components and parameters exist in both hydraulic systems and pneumatic systems. These components work together and allow the system to provide required pressure. As the future approaches technologies are evolving, and processes are becoming more sustainable. The face of the fluid power industry is changing and will continue to change. Although, the technology of hydraulic and pneumatic systems are developing; on the other hand, heat is still one of the biggest issue in a fluid power systems. The most difficult task is finding where the heat is being generated at elevated temperatures. Normal operating temperature for the majority of hydraulic systems is between $35{ }^{\circ} \mathrm{C}$ to $50{ }^{\circ} \mathrm{C}$. The usage of infrared cameras in fluid power systems can be summarized as performing regular reliability tests on a hydraulic system and locating where the heat is being generated. In addition, The cameras can be used to provide troubleshooting speed and pressure issues. There are several advantages, disadvantages for both fluid power system; and also similarities and differences exist between them. Some of them are:

Both pneumatics and hydraulic systems use fluid rather than mechanical, electrical and magnetic, or human power to transmit power from one point to another.

One of the most significant difference is that; pneumatic systems use an easily compressible gases, such as air or a suitable pure gas while hydraulic systems use relatively incompressible liquid such as oil as a working material or substance. As an advantage of that, hydraulic systems are used for more precise controlling, high force, high torque and low speed work.

Hydraulic systems can use for very high pressure applications approximately from 50 bar to 600 bar; while pneumatic systems can only use for 5 bar to 10 bar of pressure applications. Pneumatic systems use for comparatively simple controlling, low force, low torque and high speed work. So, it can be said that pneumatic systems are comparatively less efficient than hydraulic systems.

Hydraulic systems installation and maintenance is difficult also design is complex, costly and more time require. These specifications are one of the biggest disadvantages of the hydraulic systems. On the other hand, pneumatic systems installation and maintenance are comparatively very easy, less cost and less time required.

As an advantage of pneumatic systems, initial cost might be less than the cost required for a hydraulic system. However, operating cost of a pneumatic system can be five to ten times greater, compared to a hydraulic system. Because of the process of compressing atmospheric air into the nominal working pressure which requires a lot of horsepower.

\section{CKNOWLEDGMENTS}

All authors would like to thank the University of West Attica and specifically the Post Graduate Program of Studies (MSc) "New Technologies in Shipping and Transport", for the financial support provided to them to undertake this research project.

\section{REFERENCES}

[1] Gannon, M. (2018). History of pneumatics: A timeline of evolution. [online] Pneumatictips.com. Available at: https://www.pneumatictips.com/pneumatics-agestimeline-evolution-2/ [Accessed 16 Dec. 2018].

[2] 3rd Australasian Engineering Heritage Conference (2009) Remnants of Early Hydraulic Power Systems -- J W Gibson MA (Syd), MIE Aust. \& M C Pierce BE (Elec), FIE Aust.

[3] Toppr Bytes. (2018). Hydraulic Systems - Introduction, Working Principle \& more! [online] Available at: https://www.toppr.com/bytes/hydraulic-systems/ [Accessed 16 Dec. 2018].

[4] Cross MFG. (2018). Basic Hydraulic Theory | Cross Mfg.. [online] Available at: 
https://crossmfg.com/resources/technical-andterminology/basic-hydraulic-theory [Accessed 16 Dec. 2018].

[5] Anon, (2018). [ebook] Available at: https://nptel.ac.in/courses/112106175/Module\%201/Lect ure\%201.pdf [Accessed 16 Dec. 2018].

[6] Pneumatic Systems. (2018). [ebook] Available at: https://resources.hkedcity.net/ res_files/201101/20110128101153_259037.pdf [Accessed 16 Dec. 2018].

[7] Tetrapak.com. (2018). Carton packages for food and beverages. [online] Available at: https://www.tetrapak.com/rs/packaging [Accessed 16 Dec. 2018]

[8] Anon, (2018). [ebook] Available at: http://www.boschrexrothus.com/country_units/america/united_states/en/Documen tation_and_Resources/a_downloads/Air_Management_F P.pdf [Accessed 16 Dec. 2018].
[9] Machinerylubrication.com. (2019). Hydraulic Tools for Troubleshooting Any Hydraulic System. [online] Available

https://www.machinerylubrication.com/Read/3 0982/hydraulic-tools [Accessed 6 Jan. 2019].

[10] www.gembiz.co.uk, g. (2019). What does the future hold for hydraulic and pneumatic technologies? [online] Industrial technology.co.uk. Available at https://www.industrialtechnology.co.uk/products--whatdoes-the-future-hold-for-hydraulic-and-pneumatictechnologies.html [Accessed 6 Jan. 2019].

[11] YouTube. (2019). Guided tour of the Tetra Pak® A6. [online] Available at: https://www.youtube.com/watch?v=b5f6xhZ7p34\&t=17 9s\&list=WL\&index $=13$ [Accessed 7 Jan. 2019].

[12] Pepperl+Fuchs. (2019). Contrast Sensors+Color Sensors. [online] Available at: https://www.pepperlfuchs.com/global/en/classid_49.htm [Accessed 7 Jan. 2019]. 